REAL-LIFE EXPERIENCE OF OMALIZUMAB IN CHILDREN WITH SEVERE ASTHMA

${ }^{1}$ MK Koo, ${ }^{1} \mathrm{CB}$ Bossley, ${ }^{1} \mathrm{AC}$ Carlton, ${ }^{1,2} \mathrm{AB}$ Bush, ${ }^{1,2}$ SS Saglani, ${ }^{1,2} \mathrm{LF}$ Fleming; ${ }^{1}$ Department of Paediatric Respiratory Medicine, Royal Brompton Hospital, London, UK; ${ }^{2}$ National Heart and Lung Institute, Imperial College, London, UK

\subsection{6/thoraxjnl-2013-204457.238}

Introduction Therapeutic options for children with severe asthma are limited. Clinical studies support the use of the antiIgE antibody, omalizumab, in children with severe atopic asthma. However, children included in these studies had less severe disease than those in whom omalizumab is currently recommended. Little is known about the clinical efficacy of omalizumab in children with severe therapy resistant asthma (STRA).

Objectives To determine the short-term (16 weeks) and longterm (beyond 16 weeks) efficacy of omalizumab, and predictors of a successful therapeutic response in children with STRA in a clinical setting.

Methods This was an observational, prospective study of children with STRA who were commenced on omalizumab. Spirometry, bronchodilator reversibility (BDR), exhaled nitric oxide $\left(\mathrm{FE}_{\mathrm{NO}}\right)$, asthma control test (ACT), mini asthma-related quality of life questionnaire (AQLQ), severe exacerbations (requiring a course of oral corticosteroids (OCS) for $\geq 3$ days) and number of unscheduled healthcare visits (UHCV) and hospital admissions were recorded before and every 4 weeks after commencing treatment. Every 16 weeks, patients underwent a more thorough assessment to determine if the treatment should be continued.

Results 33 children (22 male) aged 5-16 years were commenced on omalizumab. At 16 weeks there were significant improvements in mini-AQLQ; ACT; $\mathrm{FE}_{\mathrm{NO}}$; maintenance OCS dose; severe exacerbations and UHCVs.

20/33 (60.6\%) children continued omalizumab beyond the initial 16 weeks (up to 192 weeks). Compared to those who discontinued, at baseline these children had higher mini-AQLQ (4.28 vs. 3.05$)$ and ACT (11 vs. 8$)$, were younger (11 vs. 13 years) and were more likely to have been admitted to hospital $(57.9 \%$ vs. $0 \%)$ and have had a severe exacerbation $(95 \%$ vs. $50 \%)$ in the 16 weeks before starting omalizumab. Maximal reduction in number of exacerbations and hospital admissions was evident at 32 weeks; this was maintained for up to 144 weeks (Figure 1).

Conclusion This is the first longitudinal study demonstrating long-term clinical efficacy of omalizumab as add-on therapy in children with STRA. Omalizumab was most effective in those with an exacerbation-prone phenotype at baseline, highlighting the importance of thorough patient characterisation when considering this treatment option.
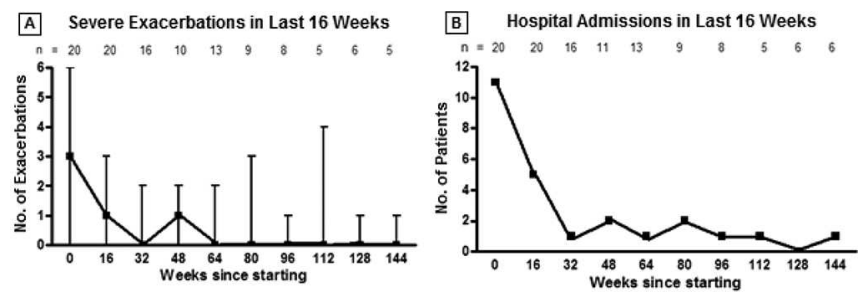

Abstract P88 Figure 1. Number of exacerbations(A) and incidence of hospital admissions (B) over timeduring treatment with omalizumab in children with severe therapy resistant asthma. Data shown is only for those who continued omalizumab beyond 16 weeks. Median and range shown for continuous data.
P89 NOVEL MECHANISMS OF IMMUNOMODULATION BY VITAMIN D AND $\alpha-1$-ANTITRYPSIN

${ }^{1}$ LV Rice, ${ }^{1} S$ Dimeloe, ${ }^{2} \mathrm{~J}$ Raynes, ${ }^{3} \mathrm{~A}$ Gupta, ${ }^{1} \mathrm{O}$ Pfeffe, ${ }^{1} \mathrm{D}$ Richards, ${ }^{1} \mathrm{Z}$ Urry, ${ }^{1} \mathrm{~S}$ Farooque, ${ }^{1} \mathrm{P}$ Ozegbe, ${ }^{1} \mathrm{E}$ Hornsby, ${ }^{4} \mathrm{M}$ Nyon, ${ }^{5} \mathrm{H}$ Haq, ${ }^{5} \mathrm{~J}$ Irving, ${ }^{1} \mathrm{~J}$ McDonnell, ${ }^{3} \mathrm{~S}$ Saglani, ${ }^{3} \mathrm{~A}$ Bush, ${ }^{4} \mathrm{~B}$ Gooptu, ${ }^{6} \mathrm{C}$ Kemper, ${ }^{1} \mathrm{C}$ Hawrylowicz; ${ }^{1} \mathrm{MRC}$ and Asthma UK Centre for Allergic Mechanisms of Asthma, King's College London, Guy's Hospital, Iondon, uk; ${ }^{2}$ Immunology and Infection Department, London School of Hygiene and Tropical Medicine, LONDON, UK; ${ }^{3}$ MRC and Asthma UK Centre for Allergic Mechanisms of Asthma, Imperial College London, Department of Paediatric Respiratory Medicine, Royal Brompton Hospital, London, UK; ${ }^{4}$ Institute of Structural and Molecular Biology/ Crystallography, Department of Biological Sciences, Birkbeck College, University of London, London, UK; ${ }^{5}$ Cambridge Institute for Medical Research, University of Cambridge, MRCNellcome Trust Building, Cambridge, UK; ${ }^{6} M R C$ Centre for Transplantation, Division of Transplantation Immunology and Mucosal Biology, King's College London, Guy's Hospital, London, UK

\subsection{6/thoraxjnl-2013-204457.239}

Vitamin D deficiency/insufficiency has been associated with poor respiratory health and a predisposition to respiratory disease. Local activation of vitamin D in the airways is important for antimicrobial defenses and suppression of inflammatory responses via the generation of a tolerogenic immune environment. One of the most highly unregulated proteins by vitamin D in CD4+ T-cells is the serine protease inhibitor $\alpha$-1-antitrypsin. In addition to controlling the pro-inflammatory effects of neutrophil elastase, $\alpha$-1-antitrypsin also acts via other pathways as an immunomodulator. We have identified a novel axis of immune modulation by vitamin $\mathrm{D}$; where $\alpha$-1-antitrypsin is able to induce IL-10 production in PBMCs via an interaction with the complement component $\mathrm{C} 3 \mathrm{a}$. Our in vitro findings are supported by in vivo correlations of serum vitamin $\mathrm{D}, \alpha$-1-antitrypsin, C3a and IL-10 in the airways of both asthmatics and healthy controls from a paediatric cohort. We propose that vitamin D is an upstream regulator of the $\alpha$-1-antitrypsin/C3a/IL-10 axis, providing attractive therapeutic options to promote tolerance in a range of inflammatory diseases.

\section{Clinical TB}

\section{P90 VITAMIN D STATUS IMPROVES FOLLOWING RECOVERY FROM TUBERCULOSIS}

${ }^{1} \mathrm{KD}$ Witt, ${ }^{1} \mathrm{DA}$ Jolliffe, ${ }^{2} \mathrm{Z}$ Wang, ${ }^{2} \mathrm{KE}$ Thummel, ${ }^{1} \mathrm{PM}$ Timms, ${ }^{1} \mathrm{CJ}$ Griffiths, ${ }^{1} \mathrm{AR}$ Martineau; ${ }^{1}$ Queen Mary University of London, London, United Kingdom; ' University of Washington, Seattle, USA

\subsection{6/thoraxjnl-2013-204457.240}

Introduction and Objectives Vitamin D deficiency associates with active tuberculosis, but the question of whether this arises as a cause or as a consequence of disease is controversial. Paired comparison of vitamin D status of TB patients at diagnosis and following recovery has potential to inform the debate, but such studies have not previously been conducted.

Methods We conducted a longitudinal study comparing serum concentrations of vitamin D metabolites in TB patients at longterm follow-up vs. diagnosis. Participants diagnosed with pulmonary TB in 2007-9 were invited to attend a follow-up visit in 2012. Concentrations of 25 -hydroxyvitamin D $(25[\mathrm{OH}] \mathrm{D}$, the measure of vitamin D status), 1 $\alpha, 25$-dihydroxyvitamin D $(1,25$ $\left.[\mathrm{OH}]_{2} \mathrm{D}\right), 24 \mathrm{R}, 25$-dihydroxyvitamin $\mathrm{D}\left(24,25(\mathrm{OH})_{2} \mathrm{D}\right), 4 \beta, 25-$ dihydroxyvitamin $\mathrm{D}\left(4,25[\mathrm{OH}]_{2} \mathrm{D}\right)$, calcium, albumin, parathyroid hormone $(\mathrm{PTH})$ and vitamin D binding protein (DBP) were determined in serum samples collected at follow-up and at the 
time of TB diagnosis. Values at the two time points were compared using Student's paired t-tests.

Results Thirty-one participants were followed up between August 2012 and February 2013. Serum 25(OH)D concentrations were significantly higher post-recovery than at diagnosis (mean 29.7 vs. $12.2 \mathrm{nmol} / \mathrm{L}, \mathrm{p}<0.0001$ ). Participants also had higher mean serum concentrations of $\mathrm{PTH}$, corrected calcium and $24,25(\mathrm{OH})_{2} \mathrm{D}$ post-recovery than at diagnosis $(\mathrm{PTH}, 4.97$ vs. $2.78 \mathrm{pmol} / \mathrm{L}, \mathrm{p}=0.0003$; corrected calcium, 2.50 vs. 2.45 $\mathrm{mmol} / \mathrm{L}, \quad \mathrm{p}=0.03 ; 24,25(\mathrm{OH})_{2} \mathrm{D}, 3.15$ vs. $1.53 \mathrm{nmol} / \mathrm{L}$, $\mathrm{p}=0.004)$. No statistically significant differences in serum concentrations of $1,25(\mathrm{OH})_{2} \mathrm{D}, 4,25(\mathrm{OH})_{2} \mathrm{D}$ or $\mathrm{DBP}$ were seen between the two time points. Differences in serum concentrations of $25(\mathrm{OH}) \mathrm{D}$ at follow-up vs. baseline remained statistically significant after exclusion of 14 participants who were taking supplemental vitamin D at follow-up and / or who had increased their sun exposure since time of diagnosis $(p=0.005)$, and after exclusion of 17 participants whose baseline sample was taken from March to July inclusive ( $\mathrm{p}=0.0003$ ).

Conclusions Vitamin D status of TB patients improved after resolution of tuberculosis. This phenomenon was not explained by differences in vitamin D supplementation, self-reported sun exposure or season of sampling at follow-up vs. baseline. Our findings raise the possibility that vitamin $\mathrm{D}$ deficiency may be a consequence, as well as a cause, of active tuberculosis.

\section{P91 INCORPORATING TUBERCULOSIS STRAIN TYPING DATA INTO ROUTINE CONTACT TRACING INVESTIGATIONS: EXPERIENCE FROM THE FIELD}

${ }^{1} \mathrm{ML}$ Munang, ${ }^{1} \mathrm{C}$ Browne, ${ }^{2} \mathrm{~S}$ Khanom, ${ }^{2} \mathrm{EG}$ Smith, ${ }^{2} \mathrm{JS}$ Evans, ${ }^{2} \mathrm{P}$ Hawkey, ${ }^{1} \mathrm{H}$ Kunst, ${ }^{1} \mathrm{~S}$ Welch, ${ }^{1} \mathrm{ML}$ Dedicoat; ${ }^{1}$ Heart of England NHS Foundation Trust, Birmingham, United Kingdom; ${ }^{2}$ Public Health England Regional Centre for Mycobacteriology, Birmingham, United Kingdom

\subsection{6/thoraxjnl-2013-204457.241}

Strain typing of tuberculosis (TB) isolates by 24 loci mycobacterial interspersed repetitive unit-variable number tandem repeats (MIRU-VNTRs) is now a routine laboratory tool for TB control, but its utility in informing contact tracing and public health action has not been well reported in the United Kingdom. Since November 2011 we have routinely held typing meetings and undertaken cluster investigation. Over 18 months, 68 clusters were discussed. Fifty-five (81\%) clusters were small (2-5 patients), 7 (10\%) were medium (6-14 patients) and 6 (9\%) were large $(>15$ patients, median $=42, \mathrm{IQR}=26-52)$. Enhanced epidemiological investigation was undertaken in $27 / 68$ (40\%) clusters. Typing meetings alone readily identified 20 definite epidemiological links between $46 / 458$ (10\%) cases. In 15 cases, 9 definite or probable links were not supported by genotyping, leading to expanded screening in one workplace. 112 extended interviews were done. A further 23 definite links between $77(17 \%)$ cases, 2 probable links between $5(1 \%)$ cases and 24 possible links between $72(16 \%)$ cases were found. Expanded screening as a direct result of strain typing and cluster investigation occurred in 4/6 settings where non-household transmission was identified (a factory, 2 places of worship, a hospital, a hostel and a pub). An additional 124 contacts were identified. 65 attended screening, 21 latent TB cases were treated and 1 active TB case was found. Routine incorporation of strain typing data in contact tracing improves diagnosis of latent and active infection but requires investment in data management systems and human resource for enhanced epidemiological investigation.

\section{P92 MILIARY TUBERCULOSIS: DATA FROM A MODERN CASE- SERIES IN THE UNITED KINGDOM}

${ }^{1} \mathrm{~N}$ Venkatraman, ${ }^{1} \mathrm{~T}$ King, ${ }^{1} \mathrm{G}$ Woltmann, ${ }^{1} \mathrm{MJ}$ Wiselka, ${ }^{1} \mathrm{D}$ Bell, ${ }^{2} \mathrm{M}$ Pareek; ${ }^{1}$ University Hospitals of Leicester, Leicester, United Kingdom; '2University of Leicester, Leicester, United Kingdom

\subsection{6/thoraxjnl-2013-204457.242}

Background Miliary tuberculosis (mTB), a severe manifestation of TB is classically associated with a high mortality. Modern data on the management and outcomes of mTB in developed world settings are lacking. We reviewed clinico-bacteriological features of mTB cases presenting to a teaching hospital in Leicester, UK serving an ethnically diverse population.

Methods Retrospective descriptive case-series of all notified mTB cases admitted between 2007 and 2012.

Results 41 cases were identified; median age 47 years (IQR:2965 years), $61 \%$ were male and $80.5 \%$ patients were of IndianSubcontinent origin. $92.5 \%$ of patients were foreign-born and median time between UK arrival and diagnosis was 4 years (IQR:1-10 years). 37(90.2\%) patients had an HIV test; 4 were positive (median CD4 count 60;IQR 30-140). Weight loss $(87.2 \%)$ and fevers $(82.9 \%)$ were the most common presenting symptoms. 30 patients $(73.2 \%)$ had abnormal examination findings; predominantly respiratory $(63.3 \%)$. Initial bloods were diagnostically nonspecific apart from lymphopaenia and depressed lymphocyte:monocyte ratio. All patients had radiological evidence of pulmonary miliary nodules. 28/41(68.3\%) patients had neuroimaging: 14/28(50\%) had neuroradiological involvement-predominantly tuberculomas (12/28-42.9\%). Lumbar punctures were undertaken in $73.2 \%$ of patients but only abnormal in 5 patients (17.2\%) (and only 1 with normal imaging). Overall, $16(39 \%)$ patients had evidence of CNS involvement.

32/41(78.1\%) patients were culture positive (all fully-sensitive) with sputum and BAL providing the highest yield. Antituberculous therapy was commenced within a median of 1 day following hospital admission. To date, 30 patients have successfully completed treatment, 3 are still on treatment, 5 have moved away and $3(7.3 \%)$ have died. In those subjects who successfully completed therapy, the lymphocyte:monocyte ratio increased significantly $(\mathrm{p}=0.0201)$. Patients who died had a longer duration between admission and commencing antituberculous treatment (median 8 days; IQR 1-16 days), than those who successfully completed treatment (median 1 day; IQR 0-3 days).

Conclusions In this developed world setting, mTB is not an uncommon presentation. Although there was a high prevalence of co-existing neurological involvement, overall mortality was low. Undertaking diagnostic procedures for culture is important and has a high yield. Early treatment may have resulted in improved outcomes and the lymphocyte:monocyte ratio may help to monitor response to treatment in miliary TB.

\section{P93 SOCIAL RISK FACTORS ASSOCIATED WITH} TUBERCULOSIS IN A HIGH INCIDENCE AREA OF THE UK 